(C) 2004 International Press

Adv. Theor. Math. Phys. 8 (2004) 987-1000

\title{
A New Infinite Class of Sasaki-Einstein Manifolds
}

\section{Jerome P. Gauntlett ${ }^{1 *}$, Dario Martelli ${ }^{2}$, James Sparks ${ }^{2}$ and Daniel Waldram ${ }^{2}$}

\author{
${ }^{1}$ Perimeter Institute for Theoretical Physics \\ Waterloo, ON, N2J 2W9, Canada \\ * On leave from: Blackett Laboratory \\ Imperial College \\ London, SW7 2AZ, UK \\ ${ }^{2}$ Blackett Laboratory, Imperial College \\ London, SW7 2AZ, UK
}

\begin{abstract}
We show that for every positive curvature Kähler-Einstein manifold in dimension $2 n$ there is a countably infinite class of associated SasakiEinstein manifolds $X_{2 n+3}$ in dimension $2 n+3$. When $n=1$ we recover a recently discovered family of supersymmetric $A d S_{5} \times X_{5}$ solutions of type IIB string theory, while when $n=2$ we obtain new supersymmetric $A d S_{4} \times X_{7}$ solutions of $D=11$ supergravity. Both are expected to provide new supergravity duals of superconformal field theories.
\end{abstract}

e-print archive:

http://lanl.arXiv.org/abs/hep-th/0403038 


\section{Introduction}

A Sasaki-Einstein manifold $X_{2 m+1}$ may be defined as a complete Riemannian manifold of dimension $2 m+1$ such that the metric cone $C\left(X_{2 m+1}\right)$ is Ricci-flat and Kähler i.e., Calabi-Yau. The restricted holonomy group of the cone is then a subgroup of $S U(m+1)$. There are various other, moreor-less equivalent, definitions. For example, $X_{2 m+1}$ may also be defined as a Riemannian manifold which admits two ${ }^{1}$ non-trivial solutions to the Killing spinor equation $\nabla_{X} \psi= \pm \frac{1}{2} \mathrm{i} X \cdot \psi$, with appropriate signs. Alternatively, the Sasaki-Einstein condition may be phrased in terms of the existence of a certain type of contact structure - indeed, this was Sasaki's definition. These conditions are all equivalent, provided $X_{2 m+1}$ is simply-connected and spin $[6]$.

There has been particular interest in Sasaki-Einstein manifolds recently due to their importance in the AdS/CFT correspondence [17]. For example, $A d S_{5} \times X_{5}$, with suitable self-dual five-form flux, is a supersymmetric solution of type IIB supergravity that is expected to be dual to a fourdimensional superconformal field theory arising from a stack of D3-branes placed at the tip of the corresponding Calabi-Yau cone $[\mathbf{1 5}, \mathbf{1 8}]$. The superconformal field theories will preserve at least $\mathcal{N}=1$ supersymmetry $[15,18,9,1]$. Similarly, $A d S_{4} \times X_{7}$, with appropriate four-form flux, is a supersymmetric solution of eleven-dimensional supergravity that is expected to be dual to a three-dimensional superconformal field theory arising on a stack of M2-branes sitting at the tip of the corresponding metric cone. The superconformal field theories will preserve at least $\mathcal{N}=2$ supersymmetry $^{2}$ $[15,18,9,1]$.

Recall that every Sasaki-Einstein manifold possesses a constant-norm Killing vector, $V$ (for some general discussion see e.g., [7]). Indeed, in the contact structure definition, $V$ is part of the geometric data. We thus have a one-dimensional foliation, and it is easy to show that the transverse geometry is locally Kähler-Einstein of positive curvature. If the orbits of $V$ close, then we have a $U(1)$ action. Since $V$ is nowhere vanishing, it follows that the isotropy groups of this action are all finite. Thus the space of leaves of the foliation will be a positive curvature Kähler-Einstein orbifold of complex dimension $m$. Such Sasaki-Einstein manifolds are called quasi-regular. If the $U(1)$ action is free, the space of leaves is actually a Kähler-Einstein

\footnotetext{
${ }^{1}$ When $m=3$ one can have weak $G_{2}$ holonomy manifolds admitting a single solution to the Killing spinor equation. In higher dimensions this is not possible - see, e.g., [6].

${ }^{2}$ If $X_{7}$ has weak $G_{2}$ holonomy, so that the metric cone has $\operatorname{Spin}(7)$ holonomy, then the $D=11$ solutions will be dual to field theories with the minimal $\mathcal{N}=1$ supersymmetry, while $\mathcal{N}=3$ theories arise as duals to tri-Sasakian $X_{7}$.
} 
manifold and the Sasaki-Einstein manifold is then said to be regular. Moreover, the converse is true: there is a Sasaki-Einstein structure on the total space of a certain $U(1)$ bundle over any given Kähler-Einstein manifold of positive curvature [16]. A similar result is true in the quasi-regular case [7]. If the orbits of $V$ do not close, the Sasaki-Einstein manifold is said to be irregular.

Although there are many results in the literature on Sasaki-Einstein manifolds in every dimension, explicit metrics are rather rare. Homogeneous regular Sasaki-Einstein manifolds are classified: they are all $U(1)$ bundles over generalised flag manifolds [6]. This result follows from the classification of homogeneous Kähler-Einstein manifolds. For example, in low dimensions with $m=1,2,3$ the homogeneous base is either $\mathbb{C} P^{1}, \mathbb{C} P^{2}, \mathbb{C} P^{1} \times \mathbb{C} P^{1}, \mathbb{C} P^{3}$, $\mathbb{C} P^{2} \times \mathbb{C} P^{1}, \mathbb{C} P^{1} \times \mathbb{C} P^{1} \times \mathbb{C} P^{1}, S U(3) / T^{2}$ or $\mathrm{Gr}_{5,2}$. The corresponding homogeneous Sasaki-Einstein manifolds are, in the physics literature, denoted $S^{3}, S^{5}, T^{1,1}, S^{7}, M^{3,2}, Q^{1,1,1}, N_{I}^{1,1}, V_{5,2}$, respectively (see for instance [8] for a review). Notice that inhomogeneous Kähler-Einstein manifolds are known to exist (including cohomogeneity one examples), and are explicit up to a function which satisfies a certain ODE - see, e.g., addendum C of [4]. One may then construct the associated regular Sasaki-Einstein manifolds.

However, until recently, there have been no known explicit inhomogeneous metrics in the quasi-regular class. Previous constructions ${ }^{3}$ of such manifolds use methods in algebraic geometry which imply existence of solutions, but do not produce explicit metrics. Moreover, no irregular examples were known to exist at all. In [10], we constructed a countably infinite family of explicit cohomogeneity one Sasaki-Einstein metrics on $S^{2} \times S^{3}$. These were discovered by dualising one of the class of M-theory solutions of [11]. This family gives not only the first explicit examples of inhomogeneous quasiregular Sasaki-Einstein manifolds, but also the first examples of irregular geometries. In this note we show that this construction extends to every dimension. More precisely, we prove the following:

For every positive curvature $2 n$-dimensional Kähler-Einstein manifold $B_{2 n}$, there is a countably infinite class of associated compact, simply-connected, spin, Sasaki-Einstein manifolds $X_{2 n+3}$ in dimension $2 n+3$. Hence each $X_{2 n+3}$ admits a Killing spinor and the holonomy of the associated metric cone is a subgroup of $S U(n+2)$. Moreover, the isometry group of $X_{2 n+3}$ is at least $G \times T^{2}$, where $G$ is the isometry group of $B_{2 n}$.

\footnotetext{
${ }^{3}$ Very recently an infinite class of explicit inhomogeneous Einstein metrics have been constructed in [14]. It is not known if any of these are Sasaki-Einstein.
} 
Note that there is also a straightforward generalisation to the case where $B_{2 n}$ is a product of Kähler-Einstein manifolds [12].

\section{The local metric}

Let $B_{2 n}$ be a (complete) $2 n$-dimensional positive curvature Kähler-Einstein manifold, with metric $d \tilde{s}^{2}$ and Kähler form $\tilde{J}$. Recall that complete Einstein manifolds with positive curvature are necessarily compact [19] and hence $B_{2 n}$ is compact. In addition, we note that a positive curvature KählerEinstein manifold is simply-connected [16]. Now, as shown in $[\mathbf{3}],[\mathbf{2 0}]$, the local metric

$$
d \hat{s}^{2}=U(\rho)^{-1} d \rho^{2}+\rho^{2} U(\rho)(d \tau-A)^{2}+\rho^{2} d \tilde{s}^{2}
$$

is then a positive curvature Kähler-Einstein metric in dimension $2 n+2$, where $2 \tilde{J}=d A$ and

$$
U(\rho)=\frac{\lambda}{2 n+2}-\frac{\Lambda}{2 n+4} \rho^{2}+\frac{\Lambda}{2(n+1)(n+2)}\left(\frac{\lambda}{\Lambda}\right)^{n+2} \frac{\kappa}{\rho^{2 n+2}}
$$

where $\kappa$ is a constant that has been rescaled compared to [20] for later convenience. The Kähler form is given by

$$
\hat{J}=\rho^{2} \tilde{J}+\rho(d \tau-A) \wedge d \rho
$$

and the curvatures of the Kähler-Einstein metrics in dimensions $2 n$ and $2 n+2$ are $\lambda>0$ and $\Lambda>0$, respectively. Thus $\widehat{\operatorname{Ric}}=\lambda \tilde{g}, \widehat{\operatorname{Ric}}=\Lambda \hat{g}$.

In $[\mathbf{2 0}]$ it was shown that the local expression (1) describes a complete metric on a manifold if and only if the base is $\mathbb{C} P^{n}$, with its canonical metric, and the resulting total space is then $\mathbb{C} P^{n+1}$, again with canonical metric. This requires the choice $\kappa=0[\mathbf{2 0}]$. For this reason, the local one-parameter family of Kähler-Einstein metrics (1) (with $\Lambda>0$ ) has been largely ignored for more than 20 years.

However, consider adding another dimension to the metric above - specifically, the local Sasaki-Einstein direction. We define the $(2 n+3)$-dimensional local metric

$$
d s^{2}=d \hat{s}^{2}+\left(d \psi^{\prime}+\sigma\right)^{2}
$$

where $d \sigma=2 \hat{J}$. As is well-known (see, e.g., [13] for a recent review), such a metric is locally Sasaki-Einstein, with curvature $2 n+2$, provided $\Lambda=2(n+2)$. An appropriate choice for the connection one-form $\sigma$ is

$$
\sigma=\frac{\lambda}{\Lambda} A+\left(\frac{\lambda}{\Lambda}-\rho^{2}\right)(d \tau-A) .
$$


We now utilise a very useful change of coordinates which casts the local metric (4) into a different $(2 n+2)+1$ decomposition. Define the new coordinates

$$
\alpha=-\tau-\frac{\Lambda}{\lambda} \psi^{\prime}
$$

and $(\Lambda / \lambda) \psi^{\prime}=\psi$. A straightforward calculation then shows that the local metric (4) assumes the following form

$$
\begin{aligned}
d s^{2}= & U(\rho)^{-1} d \rho^{2}+\rho^{2} d \tilde{s}^{2}+q(\rho)(d \psi+A)^{2} \\
& +w(\rho)[d \alpha+f(\rho)(d \psi+A)]^{2}
\end{aligned}
$$

where ${ }^{4}$

$$
\begin{aligned}
w(\rho) & =\rho^{2} U(\rho)+\left(\rho^{2}-\lambda / \Lambda\right)^{2} \\
q(\rho) & =\frac{\lambda^{2}}{\Lambda^{2}} \frac{\rho^{2} U(\rho)}{w(\rho)} \\
f(\rho) & =\frac{\rho^{2}\left(U(\rho)+\rho^{2}-\lambda / \Lambda\right)}{w(\rho)} .
\end{aligned}
$$

In order to have a Riemannian metric we must certainly ensure that $U \geq 0$, $w \geq 0, q \geq 0$. The second two conditions are in fact implied by the first, $U \geq 0$. The latter holds if we choose the range of $\rho$ to be

$$
\rho_{1} \leq \rho \leq \rho_{2}
$$

where $\rho_{i}$ are two appropriate roots of the equation $U(\rho)=0$. As we want to exclude $\rho=0$, since the metric is generically singular there, we thus take $\rho_{i}$ to be both positive (without loss of generality). Moreover, $w>0$ provided $\rho_{i}^{2} \neq \lambda / \Lambda$. If we define

$$
x=\frac{\Lambda}{\lambda} \rho^{2}
$$

we can write

$$
U(\rho)=\frac{\lambda}{2(n+1)(n+2)} \frac{1}{x^{n+1}} P(x ; \kappa)
$$

where we have introduced the polynomial in $x$

$$
P(x ; \kappa)=-(n+1) x^{n+2}+(n+2) x^{n+1}+\kappa .
$$

For any $x \geq 0$ (for fixed $\kappa$ ), a zero of $U(\rho)$ corresponds to a root of the equation $P(x ; \kappa)=0$. Observe that the only turning points of $P(x ; \kappa)$ on the interval $[0, \infty)$ are at $x=0$ and $x=1$ where the latter is a global maximum. When $\kappa$ is positive it is straightforward to see that there is only

\footnotetext{
${ }^{4}$ Note that for $n=1$ these definitions differ slightly from those in [10] when using the coordinates in section 5 .
} 
a single real root and so this is excluded. Similarly, when $\kappa<-1$ there are no real roots and this is also excluded. We thus conclude that in the range

$$
-1<\kappa \leq 0
$$

there are two distinct suitable values of the roots $\rho_{i}$

$$
0 \leq \rho_{1}<\sqrt{\frac{\lambda}{\Lambda}}<\rho_{2} \leq \sqrt{\frac{\lambda(n+2)}{\Lambda(n+1)}} .
$$

The limiting value $\kappa=0$ in (1) gives rise to a smooth compact KählerEinstein manifold if and only if $B_{2 n}=\mathbb{C} P^{n}$, in which case the resulting space is $\mathbb{C} P^{n+1}$. Clearly the corresponding Sasaki-Einstein manifold $X_{2 n+3}$, defined by (4), is $S^{2 n+3}$ (or a discrete quotient thereof). As a consequence, we now focus on the case where the range of $\kappa$ is $-1<\kappa<0$.

\section{Global analysis}

We first analyse the $(2 n+2)$-dimensional part of $(7)$, transverse to the $\alpha$ direction, and show that, with appropriate ranges and identifications of the coordinates, it is a smooth complete metric on the total space of an $S^{2}$ bundle over the original $2 n$-dimensional Kähler-Einstein manifold $B_{2 n}$. This is true for any value of the constant $\kappa$, with $-1<\kappa<0$. We will also show that the resulting $(2 n+2)$-dimensional Riemannian manifold $Y_{2 n+2}$ is in fact conformally Kähler. Secondly, we show that the $\alpha$ direction can be made into a $U(1)$ fibration over the base $Y_{2 n+2}$ for countably infinitely many values of the parameter $\kappa$ in the allowed range. The total space of the fibration is then a $(2 n+3)$-dimensional Einstein manifold. In the following section we show that it is Sasaki-Einstein.

\section{The base space $Y_{2 n+2}$}

With the range of $\rho$ being $\rho_{1} \leq \rho \leq \rho_{2}$ we see that, if $\psi$ is periodically identified, the $\rho-\psi$ part of the metric (7) at fixed point on the base $B_{2 n}$ is a circle fibred over the line segment with coordinate $\rho$, where the size of the circle goes to zero at the two roots $\rho=\rho_{i}$. This fibre will be a smooth $S^{2}$ provided the period of $\psi$ can be chosen so that there are no conical singularities where the circle collapses. Now, near a root $\rho_{i}$ we have $U(\rho) \approx U^{\prime}\left(\rho_{i}\right)\left(\rho-\rho_{i}\right)$. Defining the new coordinate

$$
R^{2}=\frac{4\left(\rho-\rho_{i}\right)}{U^{\prime}\left(\rho_{i}\right)}
$$


the $\rho-\psi$ part of the metric (7), at any fixed point on the base, is

$$
d R^{2}+\frac{\lambda^{2}}{4 \Lambda^{2}} \frac{\rho_{i}^{2} U^{\prime}\left(\rho_{i}\right)^{2}}{\left(\rho_{i}^{2}-\lambda / \Lambda\right)^{2}} R^{2} d \psi^{2}
$$

near to the root $\rho=\rho_{i}$. Now we notice the remarkable fact that

$$
\frac{\rho_{i}^{2} U^{\prime}\left(\rho_{i}\right)^{2}}{\left(\rho_{i}^{2}-\lambda / \Lambda\right)^{2}}=\Lambda^{2}
$$

for any root. Therefore the circle fibre collapses smoothly at both roots provided that $\psi$ has period $\frac{4 \pi}{\lambda}$. The $\rho-\psi$ fibre is then a smooth $S^{2}$ and hence we have a smooth complete metric on $Y_{2 n+2}$.

Now, since $d A=2 \tilde{J}$ and $\widetilde{\operatorname{Ric}}=\lambda \tilde{g}$, we see that $d A$ is $\frac{2}{\lambda}$ times the Ricci form of the base manifold $B_{2 n}$. Since the period of $\psi$ is $\frac{4 \pi}{\lambda}$, at fixed value of $\rho$ between the roots, the resulting circle fibration is just the associated $U(1)$ bundle to the canonical bundle of $B_{2 n}$. Indeed, note that one may rescale the coordinate $\rho$, as well as the other variables and coordinates, so as to set $\lambda=2$. Then the period of $\psi$ is the canonical value of $2 \pi$, and $d A$ is the Ricci form. We may therefore set

$$
\begin{aligned}
\lambda & =2 \\
\Lambda & =2(n+2)
\end{aligned}
$$

without loss of generality, where the second condition ensures that the Sasaki-Einstein metric has curvature $2(n+1)$. We will often still keep factors of $\lambda$ and $\Lambda$ in formulae, with the understanding that they take the above values.

Before studying further topological properties of these spaces, we remark that the spaces $Y_{2 n+2}$ are complex manifolds. Moreover, these manifolds are in fact conformally Kähler. Specifically, consider the fundamental two-forms

$$
J_{ \pm}=\rho^{2} \tilde{J} \pm \frac{\lambda}{\Lambda} \frac{\rho}{\sqrt{w(\rho)}} d \rho \wedge(d \psi+A)
$$

and the associated $(n+1,0)$-forms

$$
\Omega_{ \pm}=\rho^{n} \tilde{\Omega} \wedge\left[\frac{d \rho}{\sqrt{U(\rho)}} \pm i \sqrt{q(\rho)}(d \psi+A)\right]
$$

One finds that one can write $d \Omega_{ \pm}$as

$$
d \Omega_{ \pm}=L_{ \pm} \wedge \Omega_{ \pm}
$$


for suitable one-forms $L_{ \pm}$, which shows that $Y_{2 n+2}$ is complex, with complex structure specified by $\Omega_{ \pm}$. Moreover, it is immediate that

$$
d J_{ \pm}=\frac{2}{\rho}\left(1 \mp \frac{\lambda}{\Lambda} \frac{1}{\sqrt{w(\rho)}}\right) d \rho \wedge J_{ \pm}
$$

which implies that $Y_{2 n+2}$ is conformal to a Kähler manifold.

We have now constructed a complete manifold $Y_{2 n+2}$ which is the total space of an $S^{2}$ bundle over $B_{2 n}$. Let us denote the canonical bundle of $B_{2 n}$ as $\mathcal{L}$. Then $Y_{2 n+2}$ may be thought of as the manifold obtained by adding a point to each $\mathbb{C}$ fibre of $\mathcal{L}$ to obtain a $\mathbb{C} P^{1}=S^{2}$ bundle over $B_{2 n}$. We can write this bundle as $\mathcal{L} \times_{U(1)} \mathbb{C} P^{1}$. This notation means that one uses the $U(1)$ transition functions of $\mathcal{L}$ to make an associated $\mathbb{C} P^{1}$ bundle, where $U(1)$ acts isometrically on $\mathbb{C} P^{1}$ in the usual way. Notice that one can also write this as $\mathbb{P}(\mathcal{L} \oplus \mathcal{O})$, where $\mathcal{O}$ is a trivial complex line bundle over $B_{2 n}$ and $\mathbb{P}$ denotes projectivisation (i.e., one quotients out by the Hopf map $\mathbb{C}^{2} \mapsto \mathbb{C}^{2} / \mathbb{C}_{*}=\mathbb{C} P^{1}=S^{2}$ on each $\mathbb{C}^{2}$ fibre of the bundle $\left.\mathcal{L} \oplus \mathcal{O}\right)$.

We will later need a basis for the second homology group of $Y_{2 n+2}$, in terms of a basis for the base manifold $B_{2 n}$. In general we have $H_{2}\left(B_{2 n} ; \mathbb{Z}\right) \cong$ $\mathbb{Z}^{r} \oplus$ torsion, where $r$ is the rank. We will not need to know anything about the finite part (as we will be integrating two-forms over the basis). Since $\pi_{1}\left(B_{2 n}\right)$ is trivial, note that, by the universal coefficients theorem, we have $H^{2}\left(B_{2 n} ; \mathbb{Z}\right) \cong \mathbb{Z}^{r}$ is torsion-free. Now, we can find $r$ submanifolds $\Sigma_{i}, i=1, \ldots, r$, such that their homology classes $\left[\Sigma_{i}\right]$ provide a basis for the free part of the second homology group of $B_{2 n}$. Recall that $Y_{2 n+2}$ is the total space of a $\mathbb{C} P^{1}=S^{2}$ bundle over $B_{2 n}$. In fact, since this is a projectivised bundle, we can use the results of section 20 of [5] to write down the cohomology ring of $Y_{2 n+2}$ in terms of $B_{2 n}$. In particular, we note that $H^{2}\left(Y_{2 n+2} ; \mathbb{Z}\right) \cong \mathbb{Z} \oplus H^{2}\left(B_{2 n} ; \mathbb{Z}\right)$, where the generator of the first factor integrates to one over the $S^{2}$ fibre. By Poincaré duality, notice that we can find $r$ cohomology classes $\omega_{i} \in H^{2}\left(B_{2 n} ; \mathbb{Z}\right), i=1, \ldots, r$, such that $\left\langle\omega_{i},\left[\Sigma_{j}\right]\right\rangle=\delta_{i j}$. Also notice that the rank of $H_{2}\left(Y_{2 n+2} ; \mathbb{Z}\right)$ is then $r+1$. Now let $\sigma^{N}: B_{2 n} \rightarrow Y_{2 n+2}$ denote the section of $\pi: Y_{2 n+2} \rightarrow B_{2 n}$ corresponding to the "north pole" of the $S^{2}$ fibres. This is clearly a global section. Also define a submanifold $\Sigma \cong S^{2}$ of $Y_{2 n+2}$ corresponding to the fibre of $Y_{2 n+2}$ at some fixed point on the base $B_{2 n}$. Then since $\left\langle\pi^{*} \omega_{i}, \sigma_{*}^{N}\left[\Sigma_{j}\right]\right\rangle=\left\langle\omega_{i},\left[\Sigma_{j}\right]\right\rangle=$ $\delta_{i j}$, we see that $\left\{\Sigma, \sigma^{N} \Sigma_{i}\right\}$ forms a representative basis for the free part of $H_{2}\left(Y_{2 n+2} ; \mathbb{Z}\right)$.

For later use, we also note that a closed two-form $\nu$ corresponds to an integral cohomology class under the map $H^{2}\left(Y_{2 n+2} ; \mathbb{Z}\right) \rightarrow H^{2}\left(Y_{2 n+2} ; \mathbb{R}\right)$ if and only if the periods $\langle\nu,[Z]\rangle$ over our basis $Z \in\left\{\Sigma, \sigma^{N} \Sigma_{i}\right\}$ are all integral. 
We conclude this section with two technical comments. First, note that $Y_{2 n+2}$ is always a spin manifold, irrespective of whether $B_{2 n}$ is spin or not. One way to see this is to view $Y_{2 n+2}$ as the total space of the unit sphere bundle in the associated $\mathbb{R}^{3}$ bundle, $E$. Then $w_{2}(E)=w_{2}\left(\mathcal{L}_{\mathbb{R}}\right)=c_{1}(\mathcal{L})$ $\bmod 2$. But also $w_{2}\left(B_{2 n}\right)=c_{1}\left(B_{2 n}\right) \bmod 2=-c_{1}(\mathcal{L}) \bmod 2$. We may then use the Whitney formula to compute $\left.w_{2}(T E)\right|_{B_{2 n}}=w_{2}(E)+w_{2}\left(B_{2 n}\right)=$ 0 , where we view $E$ as the normal bundle to the zero section $B_{2 n}$ of $E$. Since $B_{2 n}$ is a deformation retract of the total space of $E$, this is enough to show that the total space of $E$, and therefore its boundary $Y_{2 n+2}$, are spin. Secondly, the $S^{2}$ bundle $Y_{2 n+2}$ will not in general be trivial. It was shown in [10] that if $n=1$ and $B_{2}=S^{2}$ then $Y_{4} \cong S^{2} \times S^{2}$, but this is a lowdimensional exception. Using standard techniques one can show that the first Pontryagin class of the $S O(3)$ bundle $Y_{2 n+2}$ is $c_{1}^{2}(\mathcal{L}) \in H^{4}\left(B_{2 n} ; \mathbb{Z}\right)$. In fact this can never be trivial for $n \geq 2$ since, for a Kähler-Einstein manifold $B_{2 n}, c_{1}(\mathcal{L})$ is proportional to the Kähler class $[\tilde{J}]$, and $\tilde{J}^{n}$ is proportional to the volume form, which is a non-trivial cohomology class. In particular, $c_{1}^{2}(\mathcal{L})$ must be non-trivial.

\section{The circle fibration}

We now turn to the $\alpha$ direction in the metric (7). The latter may be written

$$
d s^{2}=d s_{Y_{2 n+2}}^{2}+w(\rho)(d \alpha+B)^{2}
$$

where $B=f(\rho)(d \psi+A)$. Since for the range of $\kappa$ chosen the function $w>0$, the Killing vector $\partial / \partial \alpha$ is nowhere vanishing on the base $Y_{2 n+2}$. The idea now is that, if $\ell^{-1} B$ is a connection one-form, where $\ell \in \mathbb{R}$, then by periodically identifying $\alpha$ with period $2 \pi \ell$ we obtain a complete metric on the total space of the $U(1)$ bundle over $Y_{2 n+2}$ with connection one-form $\ell^{-1} B$. We will find that a countably infinite number of values of $\kappa$ work.

Notice that at fixed value of $\rho$ between the two roots, $B$ is proportional to the global angular form $\frac{1}{2 \pi}(d \psi+A)$ on the resulting "equatorial" $U(1)$ bundle. This is of course globally defined on this space. However, crucially, the function $f(\rho)$ does not vanish at the poles. Since the azimuthal angle $\psi$ is not defined at the poles the one-form $B$ is not well-defined on the whole manifold $Y_{2 n+2}$. However, it is straightforward to verify that $d B$ is a globally defined smooth two-form on $Y_{2 n+2}$. We now address the question of when such a closed two-form is the curvature of a connection on a complex line bundle. In fact, this is the case precisely if the periods of the two-form are all integral. Any such closed two-form $\nu$ is then the curvature of a $U(1)$ bundle (divided by $2 \pi$ ). Thus we must ensure that the periods of $\frac{1}{2 \pi} \ell^{-1} d B$ 
are integers, for some $\ell \in \mathbb{R}$. In this case, $\ell^{-1} B$ is a local connection oneform. The reason that it is not defined globally (specifically, at the poles) is that, for a non-trivial bundle, the connection $\ell^{-1} B$ is only defined in local coordinate patches, and there are gauge transformations at the intersections of the patches. The singular nature of $B$ is due to the fact that we are trying to use only one patch for a non-trivial bundle.

The periods of $\frac{1}{2 \pi} d B$ over our representative basis $\left\{\Sigma, \sigma^{N} \Sigma_{i}\right\}$ for the free part of $H_{2}\left(Y_{2 n+2} ; \mathbb{Z}\right)$ are easy to compute:

$$
\begin{gathered}
\int_{\Sigma} \frac{d B}{2 \pi}=f\left(\rho_{1}\right)-f\left(\rho_{2}\right) \\
\int_{\sigma^{N} \Sigma_{i}} \frac{d B}{2 \pi}=f\left(\rho_{2}\right) c_{(i)} .
\end{gathered}
$$

Here

$$
c_{(i)}=\int_{\Sigma_{i}} \frac{d A}{2 \pi}=\left\langle c_{1}(\mathcal{L}),\left[\Sigma_{i}\right]\right\rangle \in \mathbb{Z}
$$

are the Chern numbers. Thus we see that, if $f\left(\rho_{1}\right) / f\left(\rho_{2}\right)=p / q \in \mathbb{Q}$ is rational (with $p, q \in \mathbb{Z}$ ), then the periods of $\left[q / 2 \pi f\left(\rho_{2}\right)\right] d B$ are $\left\{p-q, q c_{(i)}\right\}$. Clearly, these are all integer. Moreover, if we set $h=\operatorname{hcf}\left\{p-q, q c_{(i)}\right\}$ then $\frac{1}{2 \pi} \ell^{-1} d B$ has integral periods with no common factor, where $\ell=h f\left(\rho_{2}\right) / q$. Notice that, using the expression (10), we have

$$
R(\kappa) \equiv \frac{f\left(\rho_{1}\right)}{f\left(\rho_{2}\right)}=\frac{\rho_{1}^{2}\left(\rho_{2}^{2}-\lambda / \Lambda\right)}{\rho_{2}^{2}\left(\rho_{1}^{2}-\lambda / \Lambda\right)}=\frac{x_{1}\left(x_{2}-1\right)}{x_{2}\left(x_{1}-1\right)}
$$

where $x_{i}$ are the associated roots of the polynomial (14). This is a continuous function of $\kappa$ in the interval $(-1,0]$. Moreover, it is easy to see that $R(0)=0$ and in the limit $R(-1)=-1$, and hence there are clearly a countably infinite number of values of $\kappa$ for which $R(\kappa)$ is rational and equal to $p / q$, with $|p / q|<1$.

In summary, this means that, for each such value of $\kappa$, since $\frac{1}{2 \pi} \ell^{-1} d B$ is a smooth globally defined two-form on $Y_{2 n+2}$ with integral periods and $w(\rho)$ is strictly positive, we can conclude that (7) defines a smooth, complete metric on $X_{2 n+3}$.

\section{The global Sasaki structure on $X_{2 n+3}$}

We denote the manifold with metric (7), and appropriate value of $\kappa$, by $X_{2 n+3}$. By construction, this is a complete, compact Einstein manifold. 
Moreover, since $X_{2 n+3}$ is the total space of a $U(1)$ fibration over a simplyconnected manifold $Y_{2 n+2}$, it is straightforward to calculate $\pi_{1}\left(X_{2 n+3}\right)$. Indeed, notice that, again by construction, the Chern numbers have no common factor, and hence it follows that $X_{2 n+3}$ is also simply-connected ${ }^{5}$. Note also that $w_{2}\left(X_{2 n+3}\right)$ is just the pull-back of $w_{2}\left(Y_{2 n+2}\right)=0$, and so $X_{2 n+3}$ is a spin manifold.

The local metric (4) that we started with is Sasaki-Einstein. The Sasaki condition means that there exists a certain contact structure. This may be phrased in terms of the existence of certain geometric objects which satisfy various algebraic and differential conditions. Specifically, the contact structure involves the Killing vector $V=\partial / \partial \psi^{\prime}$, its dual $\chi$, and the $(1,1)$ tensor constructed by raising an index of $d \chi$ with the metric. Since these objects already locally satisfy the appropriate algebraic and geometric conditions, we simply have to check that they are globally well-defined, given the identifications and ranges we have taken in order to make a complete manifold.

In terms of the coordinates (7) the unit-norm vector $V$ is easily computed to be

$$
V=\frac{\Lambda}{\lambda}\left(\frac{\partial}{\partial \psi}-\frac{\partial}{\partial \alpha}\right)
$$

This is clearly globally defined, as each of the vectors $\partial / \partial \psi, \partial / \partial \alpha$ is globally defined. Moreover, the dual one-form $\chi$ may be written

$$
\chi=\left(\rho^{2}-\frac{\lambda}{\Lambda}\right)[d \alpha+f(d \psi+A)]+\frac{\Lambda}{\lambda} q(\rho)(d \psi+A) .
$$

The function $\left(\rho^{2}-\lambda / \Lambda\right)$ is smooth on $X_{2 n+3}$, and the one-form $[d \alpha+f(d \psi+$ $A)]$ is proportional to the global angular form on the $U(1)$ fibration $U(1) \hookrightarrow$ $X_{2 n+3} \rightarrow Y_{2 n+2}$, so is globally defined on $X_{2 n+3}$. The global angular form $\frac{1}{2 \pi}(d \psi+A)$ on the "equatorial $U(1)$ bundle" contained in $Y_{2 n+2}$ at fixed $\rho$, $\rho_{1}<\rho<\rho_{2}$, is not defined at the poles of the $S^{2}$ fibres of $Y_{2 n+2}$, but the function $q(\rho)$ vanishes smoothly there, so in fact the second term is also globally defined on $X_{2 n+3}$. Clearly $\chi$ and also $d \chi$ are smooth and globally defined. We conclude that the local Sasaki structure is globally well-defined, and thus $X_{2 n+3}$ is a simply-connected spin Sasaki-Einstein manifold.

As discussed in [7], the restricted holonomy group of the metric cone on a Sasaki-Einstein manifold $X_{2 n+3}$ is a subgroup of $S U(n+2)$. Since our manifolds $X_{2 n+3}$ are simply-connected, it follows that the metric cone $C\left(X_{2 n+3}\right)$ has holonomy group contained in $S U(n+2)$, and is therefore a

\footnotetext{
${ }^{5}$ Look at the Thom-Gysin sequence for the $U(1)$ fibration $-c f$. appendix A of [10].
} 
Calabi-Yau cone. Thus, from [2], there exists a parallel spinor on $C\left(X_{2 n+3}\right)$ which projects down to a Killing spinor on $X_{2 n+3}$.

Notice that the orbits of the Killing vector $V$ close if and only if $f\left(\rho_{2}\right) \in \mathbb{Q}$. In this case the Sasaki-Einstein manifold is said to be quasi-regular, which means that the space of leaves of the foliation determined by $V$ is a KählerEinstein orbifold. Determining when $f\left(\rho_{2}\right) \in \mathbb{Q}$ seems to be a non-trivial number-theoretic problem. For the case $n=1$ studied in [10] one has to solve a quadratic diophantine, which can be done using standard methods. However, there is no general approach for solving higher-order diophantines. If $f\left(\rho_{2}\right)$ is irrational, the orbits of $V$ are dense in the torus defined by the Killing vectors $\partial / \partial \psi, \partial / \partial \alpha$. In this case the Sasaki-Einstein manifold is said to be irregular, of rank 2. There is no well-defined Kähler-Einstein base in this case. These remarks are of course consistent with [20] where it was shown that the local Kähler-Einstein metric (1) is a complete metric on a manifold only if $\kappa=0$. For the countably infinite number of values of $\kappa$ found here, the Kähler-Einstein "base" is at best an orbifold, and in the irregular case there is in fact no well-defined base at all.

\section{$5 \quad$ Connection with previous results}

If we introduce the new coordinates $\rho^{2}=(\lambda / \Lambda)(1-c y)$ and $\beta=c \tau$, the metric (1) can be cast in the following form

$$
d \hat{s}^{2}=\frac{\lambda}{\Lambda}(1-c y) d \tilde{s}^{2}+\frac{1}{F(y)} d y^{2}+\left(\frac{\lambda}{2 \Lambda}\right)^{2} F(y)(d \beta-c A)^{2}
$$

where

$$
F(y)=\frac{2 \Lambda}{(n+1)(n+2)} \frac{\left[a-y^{2} Q_{n}(1-c y)\right]}{(1-c y)^{n}}
$$

with the constant $a \equiv(\kappa+1) / c^{2}$ and $Q_{n}$ is a polynomial of degree $n$ given by

$$
Q_{n}(x)=\sum_{i=0}^{n}(i+1) x^{i} .
$$

The range of $a$, for $c=1$, is given by $0<a \leq 1$.

We are now in a position to recover the form of the five-dimensional metrics, as presented in [10]. Indeed, this is achieved by setting $n=1$, $\lambda=2$ and $\Lambda=6$ to obtain

$$
F(y)=\frac{2\left(a-3 y^{2}+2 c y^{3}\right)}{1-c y} .
$$


As a further example, we note that seven-dimensional Sasaki-Einstein metrics corresponding to $n=2, \lambda=2$ and $\Lambda=8$ have

$$
F(y)=\frac{\frac{4}{3} a-8 y^{2}+\frac{32}{3} c y^{3}-4 c^{2} y^{4}}{(1-c y)^{2}} .
$$

When $c$ is non-zero we can always choose $c=1$ by rescaling $y$. On the other hand when $c=0$ we can recover known Sasaki-Einstein metrics. Setting $c=0$, using the fact that $Q_{n}(1)=\frac{1}{2}(n+2)(n+1)$, and introducing the new coordinates $y^{2}=(2 a / \Lambda) \cos ^{2} \omega$ and $\beta^{\prime}=\left[a \lambda^{2} / 2(n+2)(n+1)\right]^{1 / 2} \beta$, we find that the metric (1) becomes

$$
d \hat{s}^{2}=\frac{\lambda}{\Lambda} d \tilde{s}^{2}+\frac{1}{\Lambda}\left[d \omega^{2}+\sin ^{2} \omega\left(d \beta^{\prime}\right)^{2}\right]
$$

which is just the direct product metric on $B_{2 n} \times S^{2}$. The corresponding Sasaki-Einstein metrics (4) are then appropriate $U(1)$ bundles over $B_{2 n} \times S^{2}$ and are clearly regular. For instance, with $n=1$ so that $B_{2}=\mathbb{C} P^{1}$, the $c=0$ limit gives the homogeneous space $T^{1,1} / \mathbb{Z}_{2}$ (or $T^{1,1}$ ) [10]. Similarly, for $n=2$, explicit metrics are obtained by taking $B_{4}$ to be $\mathbb{C} P^{1} \times \mathbb{C} P^{1}$ or $\mathbb{C} P^{2}$, and the corresponding homogeneous Sasaki-Einstein seven-manifolds are $Q^{1,1,1}$ and $M^{3,2}$, respectively. The new Sasaki-Einstein metrics in seven dimensions that we have presented here, for the special cases with $B_{4}$ given by $\mathbb{C} P^{1} \times \mathbb{C} P^{1}$ and $\mathbb{C} P^{2}$, are co-homogeneity one generalisations of $Q^{1,1,1}$ and $M^{3,2}$, respectively.

\section{Acknowledgements}

DM is funded by an EC Marie Curie Individual Fellowship under contract number HPMF-CT-2002-01539. JFS is funded by an EPSRC mathematics fellowship. DW is supported by the Royal Society through a University Research Fellowship.

\section{References}

[1] B.S. Acharya, J.M. Figueroa-O'Farrill, C.M. Hull and B. Spence, Branes at conical singularities and holography, Adv. Theor. Math. Phys. 2 (1999), 1249, hep-th/9808014.

[2] C. Bär, Real Killing spinors and holonomy, Comm. Math. Phys. 154 (1993), 509-521.

[3] L. Bérard-Bergery, Institut Elie Cartan 6 (1982), 1.

[4] A.L. Besse, Einstein Manifolds, Springer-Verlag, 2nd edition, 1987. 
[5] R. Bott and L. Tu, Differential Forms in Algebraic Topology, SpringerVerlag 1982.

[6] C.P. Boyer and K. Galicki, 3-Sasakian Manifolds, Surveys Diff. Geom. 7 (1999), 123-184, hep-th/9810250.

[7] C.P. Boyer and K. Galicki, On Sasakian-Einstein Geometry, Internat. J. Math. 11(7) (2000), 873-909, math.DG/9811098.

[8] M.J. Duff, B.E.W. Nilsson and C.N. Pope, Kaluza-Klein Supergravity, Phys. Rept. 130 (1986), 1.

[9] J.M. Figueroa-O'Farrill, Near-horizon geometries of supersymmetric branes, hep-th/9807149.

[10] J.P. Gauntlett, D. Martelli, J.F. Sparks and D. Waldram, SasakiEinstein metrics on $S^{2} \times S^{3}$, Adv. Theor. Math. Phys. 8 (2004), 711, hep-th/0403002.

[11] J.P. Gauntlett, D. Martelli, J.F. Sparks and D. Waldram, Supersymmetric $A d S_{5}$ solutions of $M$-Theory, Class. Quant. Grav. 21 (2004), 4335, hep-th/0402153.

[12] J.P. Gauntlett, D. Martelli, J.F. Sparks and D. Waldram, New AdS Solutions in String and M-Theory, to be published in 'Proceedings of the 73rd Meeting between Physicists and Mathematicians: (A)dS/CFT correspondence', 11-13 September, 2003, Strasbourg, France.

[13] G.W. Gibbons, S.A. Hartnoll and C.N. Pope, Bohm and EinsteinSasaki metrics, black holes and cosmological event horizons, Phys. Rev. D 67 (2003), 084024, hep-th/0208031.

[14] Y. Hashimoto, M. Sakaguchi and Y. Yasui, New infinite series of Einstein metrics on sphere bundles from AdS black holes, hep-th/0402199.

[15] I.R. Klebanov and E. Witten, Superconformal field theory on threebranes at a Calabi-Yau singularity, Nucl. Phys. B 536 (1998), 199, hep-th/9807080.

[16] S. Kobayashi, Topology of positively pinched Kähler manifolds, Tôhoku Math. J. 15 (1963), 121-139.

[17] J.M. Maldacena, The large $N$ limit of superconformal field theories and supergravity, Adv. Theor. Math. Phys. 2 (1998), 231 [Int. J. Theor. Phys. 38 (1999), 1113], hep-th/9711200.

[18] D.R. Morrison and M.R. Plesser, Non-spherical horizons I, Adv. Theor. Math. Phys. 3 (1999), 1, hep-th/9810201.

[19] S.B. Myers, Duke Math J. 8 (1941), 401.

[20] D.N. Page and C.N. Pope, Inhomogeneous Einstein Metrics On Complex Line Bundles, Class. Quant. Grav. 4 (1987), 213. 